\title{
The Discrete Fractional Fourier Transform
}

\author{
Çag̃atay Candan, Student Member, IEEE, M. Alper Kutay, Member, IEEE, and Haldun M. Ozaktas
}

\begin{abstract}
We propose and consolidate a definition of the discrete fractional Fourier transform that generalizes the discrete Fourier transform (DFT) in the same sense that the continuous fractional Fourier transform generalizes the continuous ordinary Fourier transform. This definition is based on a particular set of eigenvectors of the DFT matrix, which constitutes the discrete counterpart of the set of Hermite-Gaussian functions. The definition is exactly unitary, index additive, and reduces to the DFT for unit order. The fact that this definition satisfies all the desirable properties expected of the discrete fractional Fourier transform supports our confidence that it will be accepted as the definitive definition of this transform.
\end{abstract}

Index Terms-Chirplets, discrete Wigner distributions, Hermite-Gaussian functions, time-frequency analysis.

\section{INTRODUCTION}

$\mathbf{I}$ N RECENT years, the fractional Fourier transform (FRT) has attracted a considerable amount of attention, resulting in many applications in the areas of optics and signal processing. However, a satisfactory definition of the discrete FRT that is fully consistent with the continuous transform has been lacking. In this paper, our aim is to propose (following Pei et al. [1], [2]) and consolidate a definition that has the same relation with the discrete Fourier transform (DFT) as the continuous FRT has with the ordinary continuous Fourier transform. This definition has the following properties, which may be posed as requirements to be satisfied by a legitimate discrete-input/discrete-output FRT:

1) unitarity;

2) index additivity;

3) reduction to the DFT when the order is equal to unity;

4) approximation of the continuous FRT.

The first two are essential properties of the continuous transform, which we desire to be satisfied exactly by the discrete transform. The third is necessary for the discrete fractional Fourier transform to be a consistent generalization of the ordinary DFT. The last, of course, is the major motivation for defining the discrete transform in the first place. Beyond these, it would be desirable for the discrete transform to satisfy as many operational properties of the continuous transform as possible.

Manuscript received December 1, 1998; revised October 4, 1999. The associate editor coordinating the review of this paper and approving it for publicaiton was Dr. Phillip A. Regalia.

Ç. Candan was with the Department of Electrical Engineering, Bilkent University, Ankara, Turkey. He is now with the School of Electrical and Computer Engineering, Georgia Institute of Technology, Atlanta, GA 30032 USA.

M. A. Kutay was with the Department of Electrical Engineering, Bilkent University, Ankara, Turkey. He is now with the Department of Electrical and Computer Engineering, Drexel University, Philadelphia, PA 19104 USA.

H. M. Ozaktas is with the Department of Electrical Engineering, Bilkent University, Ankara, Turkey.

Publisher Item Identifier S 1053-587X(00)03334-1.
A comprehensive introduction to the FRT and historical references may be found in [5]. The transform has become popular in the optics and signal processing communities following the works of Ozaktas and Mendlovic [6]-[8], Lohmann [9] and Almeida [12]. Some of the applications explored include optimal filtering in fractional Fourier domains [13]-[16], cost-efficient linear system synthesis and filtering [17]-[21], time-frequency analysis [11], [12], [22], [23], and Fourier optics and optical information processing [24]-[26]. Additional recent publications include [27]-[33]. Further references may be found in [5].

Up to now, the fractional Fourier transform has been digitally computed using a variety of approaches. However, these approaches are often far from exhibiting the internal consistency and analytical elegance we take for granted with the ordinary DFT. It is the purpose of this paper to offer and consolidate such a definition of the discrete fractional Fourier transform.

A fast $O(N \log N)$ algorithm for digitally computing the continuous fractional Fourier transform integral has been given in [34]. This method maps the $N$ samples of the original function to the $N$ samples of the transform. Whereas this mapping is very satisfactory in terms of accuracy, the $N \times N$ matrix underlying this mapping is not exactly unitary and does not exactly satisfy the index additivity property. This makes it unsuitable for a self-consistent a priori definition of the discrete transform.

Several publications proposing a definition for the discrete FRT have appeared, but none of these papers satisfy all the requirements. Most of these provide a satisfactory approximation to the continuous transform; however, [34] and [35] do not satisfy requirements 1 and 2, and [36] does not satisfy requirement 3. Reference [37] satisfies requirement 2 for certain discrete orders, and it is not clear to us whether it satisfies requirement 4 . The definition in [38] and [39] corresponds to a completely distinct definition of the fractional Fourier transform [40].

The definition proposed in this paper was first suggested by Pei and Yeh [1], [2]. They suggest defining the discrete FRT in terms of a particular set of eigenvectors (previously discussed in [38]), which they claim to be the discrete analogs of the Hermite-Gaussian functions (which are well known as the eigenfunctions of the continuous transform). They also justify their claims by numerical observations and simulations. In the present paper, we provide an analytical development of Pei's claims with the aim of consolidating the definition of the discrete FRT.

In Section II, the definition and some properties of the continuous FRT are presented. In Section III, the definition of the discrete fractional Fourier transform is given. Certain extensions are provided in Section IV. The paper concludes with numerical comparisons and future research directions. 


\section{PRELiminaries}

\section{A. Continuous Fractional Fourier Transform}

The $a$ th-order continuous FRT can be defined for $0<|a|<2$ through its integral kernel

$$
\begin{aligned}
\left\{\mathcal{F}^{a} f\right\}\left(t_{a}\right) & =\int_{-\infty}^{\infty} K_{a}\left(t_{a}, t\right) f(t) d t \\
K_{a}\left(t_{a}, t\right) & =K_{\phi} e^{j \pi\left(t_{a}^{2} \cot \phi-2 t_{a} t \csc \phi+t^{2} \cot \phi\right)}
\end{aligned}
$$

where $\phi=a \pi / 2$ and $K_{\phi}=\exp [-j(\pi \operatorname{sgn}(\phi) / 4-$ $\phi / 2)] /|\sin (\phi)|^{0.5}$. The kernel $K_{a}\left(t_{a}, t\right)$ is defined separately for $a=0$ and $a= \pm 2$ as $K_{0}\left(t_{a}, t\right)=\delta\left(t_{a}-t\right)$ and $K_{ \pm 2}\left(t_{a}, t\right)=\delta\left(t_{a}+t\right)$. The definition can easily be extended outside the interval $[-2,2]$ by noting that $\mathcal{F}^{4 l+a} f\left(t_{a}\right)=\mathcal{F}^{a} f\left(t_{a}\right)$ for any integer $l$. The kernel is known to have the following spectral expansion [3]:

$$
K_{a}\left(t_{a}, t\right)=\sum_{k=0}^{\infty} \psi_{k}\left(t_{a}\right) e^{-j \frac{\pi}{2} k a_{2}} \psi_{k}(t)
$$

where $\psi_{k}(t)$ denotes the $k$ th Hermite-Gaussian function, and $t_{a}$ denotes the variable in the ath-order fractional Fourier domain. Here, $\exp (-j \pi k a / 2)$ is the $a$ th power of the eigenvalue $\lambda_{k}=$ $\exp (-j \pi k / 2)$ of the ordinary Fourier transform. When $a=1$, the FRT reduces to the ordinary Fourier transform $\{\mathcal{F} f\}\left(t_{1}\right)=$ $\int e^{-j 2 \pi t_{1} t} f(t) d t$, where $t_{1}$ denotes the frequency-domain variable. As $a$ approaches zero or integer multiples of \pm 2 , the kernel approaches $\delta\left(t_{a}-t\right)$ and $\delta\left(t_{a}+t\right)$, respectively [4]. The most important properties of the FRT are

1) unitarity: $\left(\mathcal{F}^{a}\right)^{-1}=\mathcal{F}^{-a}=\left(\mathcal{F}^{a}\right)^{\dagger}$, where $(\cdot)^{\dagger}$ denotes Hermitian conjugation;

2) index additivity: $\mathcal{F}^{a_{1}} \mathcal{F}^{a_{2}}=\mathcal{F}^{a_{2}} \mathcal{F}^{a_{1}}=\mathcal{F}^{a_{1}+a_{2}}$;

3) reduction to the ordinary Fourier transform when $a=1$. Another important property not discussed here is the relationship of the fractional Fourier transform to time-frequency representations such as the Wigner distribution [8], [10], [11], [12].

We will define the discrete FRT through a discrete analog of (2). Therefore, we will first discuss the Hermite-Gaussian functions in some detail.

\section{B. The Hermite-Gaussian Functions}

The $k$ th-order Hermite-Gaussian function is defined as $(k=$ $0,1, \ldots)$

$$
\psi_{k}(t)=\frac{2^{1 / 4}}{\sqrt{2^{k} k !}} H_{k}(\sqrt{2 \pi} t) e^{-\pi t^{2}}
$$

where $H_{k}$ is the $k$ th Hermite polynomial having $k$ real zeros. The Hermite-Gaussians form a complete and orthonormal set in $\mathcal{L}_{2}$ [42]. The Hermite-Gaussian functions are well known to be the eigenfunctions of the Fourier transform operator, as will also be seen below.

We begin with the defining differential equation of the Hermite-Gaussians:

$$
\frac{d^{2} f(t)}{d t^{2}}-4 \pi^{2} t^{2} f(t)=\lambda f(t)
$$

It can be shown that the Hermite-Gaussian functions are the unique finite energy eigensolutions of (4) (see [43, p. 337]). We can express the left-hand side of (4) in abstract operator notation as

$$
\left(\mathcal{D}^{2}+\mathcal{F} \mathcal{D}^{2} \mathcal{F}^{-1}\right) f(t)=\lambda f(t)
$$

where $\mathcal{D}=d / d t$ and $\mathcal{F}$ denote the differentiation and the ordinary Fourier transform operations, respectively. The operator $\left(\mathcal{D}^{2}+\mathcal{F} \mathcal{D}^{2} \mathcal{F}^{-1}\right)$ can also be recognized as the Hamiltonian associated with the quantum-mechanical harmonic oscillator [44].

Here, we will denote this operator by $\mathcal{S}$ and thus write (5) as

$$
\left(\mathcal{D}^{2}+\mathcal{F} \mathcal{D}^{2} \mathcal{F}^{-1}\right) f(t)=\mathcal{S} f(t)=\lambda f(t) .
$$

A theorem of commuting operators will be used to show that the Hermite-Gaussian functions, which are eigenfunctions of $\mathcal{S}$, are also eigenfunctions of $\mathcal{F}$ (see [45, p. 52]).

Theorem 1: If two operators $\mathcal{A}$ and $\mathcal{B}$ commute, i.e., $\mathcal{A B}=$ $\mathcal{B} \mathcal{A}$, there exists a common eigenvector set between $\mathcal{A}$ and $\mathcal{B}$.

The commutation of $\mathcal{F}$ and $\mathcal{S}$ can be shown as

$$
\begin{aligned}
\mathcal{F S} & =\mathcal{F} \mathcal{D}^{2}+\mathcal{F}^{2} \mathcal{D}^{2} \mathcal{F}^{-1}=\mathcal{F} \mathcal{D}^{2}+\mathcal{F}^{2} \mathcal{D}^{2} \mathcal{F}^{-2} \mathcal{F} \\
& =\mathcal{F} \mathcal{D}^{2}+\mathcal{D}^{2} \mathcal{F}=\mathcal{S F} .
\end{aligned}
$$

In passing from the third term to the fourth, we used $\mathcal{F}^{2} \mathcal{D}^{2} \mathcal{F}^{-2}=\mathcal{J} \mathcal{D}^{2} \mathcal{J}=\mathcal{D}^{2}$. This, in turn, follows from the fact that $\mathcal{F}^{2}=\mathcal{F}^{-2}=\mathcal{J}$ (where $\mathcal{J} f(t)=f(-t)$ ). Thus, this proves that the Hermite-Gaussian functions, which are the unique finite energy eigenfunctions of $\mathcal{S}$, are also eigenfunctions of $\mathcal{F}$.

\section{DISCRETE FRACTIONAL FOURIER TRANSFORM}

We will first show that the first three requirements are automatically satisfied when the fractional transform is defined through a spectral expansion analogous to (2). Assuming $p_{k}[n]$ to be an arbitrary orthonormal eigenvector set of the $N \times N$ DFT matrix and $\lambda_{k}$ to be the associated eigenvalues, the discrete analog of (2) is

$$
\mathbf{F}^{a}[m, n]=\sum_{k=0}^{N-1} p_{k}[m]\left(\lambda_{k}\right)^{a} p_{k}[n]
$$

which constitutes a definition of the discrete fractional Fourier transform matrix $\mathbf{F}^{a}$. This transform matrix is unitary since the eigenvalues $\lambda_{k}=\exp (-j \pi k / 2)$ of the DFT matrix have unit magnitude [38], [41]. Reduction to the DFT when $a=1$ follows from the fact that when $a=1$, (8) reduces to the spectral expansion of the ordinary DFT matrix. Index additivity can likewise be easily demonstrated by multiplying the matrices $\mathbf{F}^{a_{1}}$ and $\mathbf{F}^{a_{2}}$ and using the orthonormality of the $p_{k}[n]$ [48]. Additionally, it is easy to show that any definition satisfying these three requirements can always be expressed in the spectral expansion form.

Before we continue, we note that there are two ambiguities that must be resolved in (8). The first concerns the eigenstructure of the DFT. Since the DFT matrix has only four distinct eigenvalues $\left(\lambda_{k}=\exp (-j \pi k / 2) \in\{1,-1, j,-j\}\right)$ [41], the eigenvalues are in general degenerate so that the eigenvector set 
is not unique. For this reason, it is necessary to specify a particular eigenvector set to be used in (8). In the continuous case, this ambiguity is resolved by choosing the Hermite-Gaussian functions as the eigenfunctions or, equivalently, by choosing that eigenfunction set of the Fourier transform that are also eigenfunctions of $\mathcal{S}$. In other words, we choose the common eigenfunction set of the commuting operators $\mathcal{S}$ and $\mathcal{F}$. Since our aim is to obtain a definition of the discrete transform that is completely analogous to the continuous transform, we will resolve this ambiguity in the same manner by choosing the common eigenvector set of the DFT matrix and the discrete matrix analog of $\mathcal{S}$, which we define to be the discrete counterparts of the Hermite-Gaussian functions.

The second ambiguity arises in taking the fractional power of the eigenvalues since the fractional power operation is not single valued. This ambiguity will again be resolved by analogy with the continuous case by taking $\lambda_{k}^{a}=\exp (-i \pi k a / 2)$. Distinct definitions based on other choices are discussed in [40]. The particular choice we are concentrating on is the one that has been most studied and has overwhelmingly found the largest number of applications.

Denoting the discrete Hermite-Gaussians as $u_{k}[n]$, the definition of the discrete fractional Fourier transform becomes

$$
\mathcal{F}^{a}[m, n]=\sum_{k=0}^{N-1} u_{k}[m] e^{-j \frac{\pi}{2} k a} u_{k}[n] .
$$

Now, we must explicitly define the discrete counterparts of the Hermite-Gaussian functions.

\section{A. Discrete Hermite-Gaussians}

We will define the discrete Hermite-Gaussians as solutions of a difference equation that is analogous to the defining differential (4) of the continuous Hermite-Gaussian functions. First, we define the second difference operator $\tilde{\mathcal{D}}^{2}$

$$
\tilde{\mathcal{D}}^{2} f(t)=\frac{f(t+h)-2 f(t)+f(t-h)}{h^{2}}
$$

which serves as an approximation to $\mathcal{D}^{2}=d^{2} / d t^{2}$. This can also be seen by examining

$$
\begin{aligned}
\tilde{\mathcal{D}}^{2} & =\frac{e^{h \mathcal{D}}-2+e^{-h \mathcal{D}}}{h^{2}} \\
& =\underbrace{\mathcal{D}^{2}+\underbrace{\frac{2 h^{2}}{4 !} \mathcal{D}^{4}+\frac{2 h^{4}}{6 !} \mathcal{D}^{6}+\cdots}}_{O\left(h^{2}\right)}
\end{aligned}
$$

where we have expressed the shift operator in hyperdifferential form as $f(t+h)=e^{h \mathcal{D}} f(t)$ [46].

Now, we consider the finite difference analog of $\mathcal{F} \mathcal{D}^{2} \mathcal{F}^{-1}$ appearing in (6), which is $\mathcal{F} \tilde{\mathcal{D}}^{2} \mathcal{F}^{-1}$.

$$
\begin{aligned}
\mathcal{F} \tilde{\mathcal{D}}^{2} \mathcal{F}^{-1} & =\mathcal{F}\left[\frac{e^{h \mathcal{D}}-2+e^{-h \mathcal{D}}}{h^{2}}\right] \mathcal{F}^{-1} \\
& =\frac{e^{j 2 \pi h t}-2+e^{-j 2 \pi h t}}{h^{2}}=\frac{2(\cos (2 \pi h t)-1)}{h^{2}} \\
& =-4 \pi^{2} t^{2}+\frac{h^{2}}{12}\left(16 \pi^{4} t^{4}\right)+O\left(h^{4}\right)
\end{aligned}
$$

where we used the fact that $\mathcal{F}^{h \mathcal{D} \mathcal{F}^{-1}}=e^{j 2 \pi h t}$, which is nothing but a statement of the shift property of the ordinary Fourier transform.

Finally, we replace $\mathcal{D}^{2}$ in (6) with $\tilde{\mathcal{D}}^{2}$ to obtain an approximation of $\mathcal{S}$, which we refer to as $\tilde{\mathcal{S}}$

$$
\begin{aligned}
\tilde{\mathcal{S}} & =\tilde{\mathcal{D}}^{2}+\mathcal{F} \tilde{\mathcal{D}}^{2} \mathcal{F}^{-1} \\
& =\frac{e^{h \mathcal{D}}-2+e^{-h \mathcal{D}}}{h^{2}}+\frac{2(\cos (2 \pi h t)-1)}{h^{2}} \\
& =\mathcal{D}^{2}-4 \pi t^{2}+\frac{h^{2}}{12}\left(\mathcal{D}^{4}+16 \pi^{4} t^{4}\right)+O\left(h^{4}\right) .
\end{aligned}
$$

We see that the analogous finite difference operator $\tilde{\mathcal{S}}$ is an $O\left(h^{2}\right)$ approximation of $\mathcal{S}$. If we explicitly write the difference equation $\tilde{\mathcal{S}} f(t)=\lambda f(t)$, we obtain

$$
\begin{aligned}
& f(t+h)-2 f(t)+f(t-h) \\
& \quad+2(\cos (2 \pi h t)-1) f(t)=h^{2} \lambda f(t) .
\end{aligned}
$$

We will now switch to discrete variables by letting $t=n h$ [46] and obtain the second-order difference equation analogous to the defining differential equation of Hermite-Gaussians as

$$
\begin{aligned}
& f[n+1]-2 f[n]+f[n-1] \\
& \quad+2\left(\cos \left(\frac{2 \pi}{N} n\right)-1\right) f[n]=\lambda f[n]
\end{aligned}
$$

where $f[n]=f(n h)$, and $h=1 / \sqrt{N}$. We immediately note that the coefficients of the above equation are periodic with $N$, implying the existence of periodic solutions with the same period [47]. When (15) is written explicitly by concentrating on a single period, say, $0 \leq n \leq N-1$, we obtain (16), shown at the bottom of the next page, where the rows of (16) follow from the replacement of $0 \leq n \leq N-1$ in (15) and the utilization of the periodicity relation $f[n+N]=f[n]$. This completes the derivation of the discrete analog of $\mathcal{S}$, which we will refer to as the $\mathbf{S}$ matrix.

We will show below that $\mathbf{S}$ commutes with the DFT matrix and that the common eigenvector set of $\mathbf{S}$, as well as the DFT matrix, is unique and orthogonal.

This unique orthogonal eigenvector set, which we call $\mathbf{u}_{k}$, will be taken as the discrete counterpart of the continuous Hermite-Gaussians to be used in the defining (9).

We now demonstrate that the $\mathbf{S}$ and the DFT matrices commute.

Theorem 2: The matrix $\mathbf{S}$ and the DFT matrix (F) commute.

Proof: $\mathbf{S}$ can be written as $\mathbf{S}=\mathbf{A}+\mathbf{B}$, where $\mathbf{A}$ is the circulant matrix corresponding to the system whose impulse response is $h[n]=\delta[n+1]-2 \delta[n]+\delta[n-1]$, and $\mathbf{B}$ is the diagonal matrix defined as $\mathbf{B}=\mathbf{F A F}^{-1}$. It can also be seen that $\mathbf{F B F} \mathbf{F}^{-1}=\mathbf{F}^{2} \mathbf{A} \mathbf{F}^{2}=\mathbf{A}$ since $h[n]$ is an even function. Then, $\mathbf{F S F}^{-1}=\mathbf{F}(\mathbf{A}+\mathbf{B}) \mathbf{F}^{-1}=\mathbf{B}+\mathbf{A}=\mathbf{S}$.

In the next subsection, we will show that the common eigenvector set is unique, and in the following subsection, we will discuss the issue of ordering (or indexing) of the eigenvectors in one-to-one correspondence with the continuous Hermite-Gaussian functions. 


\section{B. Uniqueness of the Common Eigenvector Set of $\mathbf{S}$ and DFT}

The eigenvectors of the DFT matrix are either even or odd vectors [41]. It follows from this result that the common eigenvector set of $\mathbf{S}$ and the DFT matrix, which is known to exist since they commute, should also consist of even or odd vectors. For completeness, we present a short proof of this important result, which will be utilized in developments below (see also [41] for an alternative proof).

Theorem 3: Eigenvectors of the DFT matrix are either even or odd sequences.

Proof: Letting $\mathbf{F}$ denote the DFT matrix, we know that $\mathbf{F}^{2}=\mathbf{J}$ and $\mathbf{F}^{4}=\mathbf{I}$, where $\mathbf{J}$ is the coordinate inversion matrix, and $\mathbf{I}$ is the identity matrix. Since $\mathbf{F}^{4}=\mathbf{I}$, the eigenvalues $\lambda$ of $\mathbf{F}$ can only be $\{\mathbf{1},-1, j,-j\}$. Now, let $\mathbf{p}$ be an eigenvector of $\mathbf{F}$ satisfying $\mathbf{F} \mathbf{p}=\lambda \mathbf{p}$; then, $\mathbf{F}^{2} \mathbf{p}=\lambda^{2} \mathbf{p}$; however, since $\lambda \in\{\mathbf{1}, j,-j,-\mathbf{1}\}, \mathbf{F}^{2} \mathbf{p}=\lambda^{2} \mathbf{p}$ is equivalent to $\mathbf{J} \mathbf{p}= \pm \mathbf{p}$, and that completes the proof.

It is known that when $N$ is not a multiple of 4 , all of the eigenvalues of $\mathbf{S}$ are distinct [38]. Since $\mathbf{S}$ is a real symmetric matrix, it follows that all of its eigenvectors are orthogonal to each other, and thus, the set of eigenvectors of $\mathbf{S}$ is orthogonal and unique (within multiplicative constants). Since we have already shown that $\mathbf{S}$ has a common eigenvector set with the DFT matrix, this unique set of eigenvectors of $\mathbf{S}$ must also be a set of eigenvectors of the DFT matrix. The normalized version of this set of eigenvectors will be defined as the discrete version of the Hermite-Gaussian functions.

When $N$ is a multiple of 4 , the matrix $\mathbf{S}$ still has distinct eigenvalues, with the exception of one eigenvalue, which has the value of zero with degeneracy two. The eigenvectors corresponding to all eigenvalues except this one are orthogonal to each other. The two eigenvectors corresponding to the zero eigenvalue can be chosen to be orthogonal, again because $\mathbf{S}$ is a real symmetric matrix. There are many ways of choosing these two eigenvectors such that they are orthogonal; however, there is only one way to choose them such that one is an even vector and one is an odd vector. Since we are seeking the common set of eigenvectors between $\mathbf{S}$ and the DFT matrix and since we know that all eigenvectors of the DFT matrix are either even or odd vectors, we have no choice but to choose the even and odd eigenvectors corresponding to the zero eigenvalue; other choices could not be an eigenvector of the DFT matrix. This requirement resolves the ambiguity associated with choosing the eigenvectors corresponding to the zero eigenvalue when $N$ is a multiple of 4 and again uniquely determines the common set of eigenvectors of $\mathbf{S}$ and the DFT matrix.

We have seen that the common eigenvector set has to be formed by even and odd vectors. Therefore, we will restrict the search for the common set on the even and the odd spaces. To do that, we will introduce a matrix $\mathbf{P}$ that decomposes an arbitrary vector $f[n]$ into its even and odd components. The $\mathbf{P}$ matrix, as defined below, maps the even part of the $N$-dimensional vector $f[n]$ to the first $\lfloor(N / 2+1)\rfloor$ components and the odd part to the remaining components. ${ }^{1}$ For example, the $\mathbf{P}$ matrix of dimension 5 is

$$
P=\frac{1}{\sqrt{2}}\left[\begin{array}{ccccc}
\sqrt{2} & 0 & 0 & 0 & 0 \\
0 & 1 & 0 & 0 & 1 \\
0 & 0 & 1 & 1 & 0 \\
0 & 0 & 1 & -1 & 0 \\
0 & 1 & 0 & 0 & -1
\end{array}\right] .
$$

The first three components of $\mathbf{g}=\mathbf{P f}$

$$
\begin{aligned}
{[g[0], g[1], g[2]] } & =\frac{1}{\sqrt{2}}[\sqrt{2} f[0], f[1]+f[-1], f[2]+f[-2]] \\
& =\frac{1}{\sqrt{2}}[\sqrt{2} f[0], f[1]+f[4], f[2]+f[3]]
\end{aligned}
$$

represent the components of the even part of $f[n]$. Similarly, the remaining two components represents the odd part of $f[n]$. We remember that the arguments are interpreted modulo $N$, as in the study of the ordinary DFT. In addition, note that the $\mathbf{P}$ matrix is both symmetric and unitary, that is, $\mathbf{P}=\mathbf{P}^{T}=\mathbf{P}^{-1}$.

If we consider the similarity transformation $\mathbf{P S P}^{-1}$, we expect the resultant matrix to be in the block diagonal form, that is

$$
\mathbf{P S P}^{-1}=\mathbf{P S P}=\left[\begin{array}{cc}
\mathbf{E v} & 0 \\
0 & \mathbf{O d}
\end{array}\right] .
$$

Otherwise, the $\mathbf{S}$ matrix cannot have all even/odd eigenvector set. It is clearly seen that eigenvectors of $\mathbf{P S P}^{-1}$ can be determined separately from Ev and Od matrices, and the corresponding eigenvectors of $\mathbf{S}$ are simply the even/odd extension of

${ }^{1}\lfloor x\rfloor$ is the greatest integer less than or equal to the argument.

$$
\begin{aligned}
& {\left[\begin{array}{cccclc}
-2 & 1 & 0 & \ldots & 0 & 1 \\
1 & 2 \cos \left(\frac{2 \pi}{N}\right)-4 & 1 & \ldots & 0 & 0 \\
0 & 1 & 2 \cos \left(\frac{2 \pi}{N} 2\right)-4 & \ldots & 0 & 0 \\
\vdots & \vdots & \vdots & \ddots & \vdots & \vdots \\
1 & 0 & 0 & \ldots & 1 & 2 \cos \left(\frac{2 \pi}{N}(N-1)\right)-4
\end{array}\right]} \\
& \times\left[\begin{array}{c}
f[0] \\
f[1] \\
f[2] \\
\vdots \\
f[N-2] \\
f[N-1]
\end{array}\right]=\lambda\left[\begin{array}{c}
f[0] \\
f[1] \\
f[2] \\
\vdots \\
f[N-2] \\
f[N-1]
\end{array}\right]
\end{aligned}
$$


the eigenvectors of $\mathbf{P S P}^{-1}$. Therefore, the problem of finding the common eigenvector set is reduced to finding the eigenvectors of the Ev and Od matrices.

The $\mathbf{S}$ matrix has a tridiagonal structure, except the two entries at the upper-right and lower-left corners. After the similarity transformation, $\mathbf{P S P}^{-1}$ becomes exactly tri-diagonal, meaning that the submatrices $\mathbf{E v}$ and $\mathbf{O d}$ are also tridiagonal. From [45], we know that tridiagonal matrices have distinct eigenvalues, and this result implies the uniqueness of the eigenvectors of the $\mathbf{E v}$ and $\mathbf{O d}$ matrices and completes the proof of the uniqueness of the common eigenvector set of $\mathbf{S}$ and the DFT matrix.

Note that in [38], the matrix $\mathbf{S}+4 \mathbf{I}$ is employed as a vehicle to obtain an orthogonal eigenvector set for the DFT matrix. The authors of [38] conjecture that the eigenvalues of $\mathbf{S}+4 \mathbf{I}$ are distinct whenever the dimension of $\mathbf{S}+4 \mathbf{I}$ is not a multiple of 4. In the present paper, we show that the eigenvalue degeneracy of $\mathbf{S}$ is at most two, and even though there exist many ways of choosing these two orthogonal eigenvectors, there is only one way to choose them such that one is an even vector and other is an odd vector. This argument uniquely determines the common set of eigenvectors of $\mathbf{S}$ and the DFT matrix, whatever the dimension of $\mathbf{S}$.

\section{Ordering the Eigenvectors of $\mathbf{S}$}

In the previous sections, we have shown the existence and uniqueness of the common eigenvector set of $\mathbf{S}$ and the DFT matrix. In this section, we will determine which eigenvector of this set corresponds to which Hermite-Gaussian function. We will order (index) the eigenvector set in a manner consistent with the ordering of the continuous Hermite-Gaussians. Our method of ordering will be based on the zero-crossings of the discrete Hermite-Gaussians, in analogy with the zeros of the continuous Hermite-Gaussians.

We will first clarify what we mean by the zero crossing of a discrete vector. The vector $f[n]$ has a zero crossing at $n$ if $f[n] f[n+1]<0$. In counting the number of zero crossings of the periodic sequence $f[n]$ with period $N$, we count the number of zeros in the period $0 \leq n \leq N-1$, where we also include the zero crossing at the endpoints of the period such as when $f[N-1] f[N]=f[N-1] f[0]<0$ [48]. With this convention, it can be seen that the shifted periodic sequences have the same number of zero crossings, regardless of the shift. Therefore, the number of zero crossings becomes a property of the periodic sequences and not just a property of a particular period.

Now, we need to show that all eigenvectors have a distinct number of zero crossings and need to establish a convenient method for counting the zero crossings. The exhaustive counting of the zero crossings can be numerically problematic due to the difficulty of determining the sign of a component which is of small magnitude.

To find the eigenvector $\mathbf{u}_{k}$ with $k$ zero crossings, we will combine two results from [45]. As discussed before, the common eigenvectors of $\mathbf{S}$ and the DFT matrix can be derived from the eigenvectors of the tridiagonal $\mathbf{E v}$ and $\mathrm{Od}$ matrices. An explicit expression for the eigenvectors of tridiagonal matrices is given in [45, p. 316]. Combining this expression with the Sturm sequence theorem [45, p. 300], we can show that the eigenvector of the Ev or Od matrix with the highest eigenvalue has no zero crossings, the eigenvector with the second highest eigenvalue has one zero crossing, and so on [48]. Therefore, we can show that the Ev and Od matrices have eigenvectors whose number of zero crossings range from 0 to $\lfloor N / 2\rfloor$ and from 0 to $\lfloor(N-3) / 2\rfloor$, respectively [48].

Since the even and odd eigenvectors of $\mathbf{S}$ are derived from the zero-padded eigenvectors of $\mathbf{E v}$ and $\mathbf{O d}$, we can show that after zero padding and transformation through $\mathbf{P}$, which is nothing but even/odd extension of the vector, the eigenvector of $\mathbf{E v}$ with $k$ zero crossings yields the even eigenvector of $\mathbf{S}$ with $2 k(0 \leq$ $k \leq\lfloor N / 2\rfloor)$ zero crossings, and the eigenvector of $\mathrm{Od}$ with $k$ zero crossings yields the odd eigenvector of $\mathbf{S}$ with $2 k+1$ $(0 \leq k \leq\lfloor(N-3) / 2\rfloor)$ zero crossings. This eigenvalue-based procedure enables us to accurately determine the number of zero crossings of an eigenvector without employing any means of counting. [48].

An even eigenvector of $\mathbf{S}$ can be formed as $\mathbf{e}=$ $\mathbf{P}\left[\hat{\mathbf{e}}_{k}^{T}: 0 \ldots 0\right]^{T}$, where $\hat{\mathbf{e}}_{k}$ is the eigenvector of $\mathbf{E v}$ with $k$ zero-crossings $(0 \leq k \leq\lfloor N / 2\rfloor)$. It can be seen at this point that the vector $\mathbf{e}$

$$
\mathbf{e}=\frac{1}{\sqrt{2}}\left[\sqrt{2} \hat{e}_{k}[0] \hat{e}_{k}[1] \ldots \hat{e}_{k}[r]: \hat{e}_{k}[r] \ldots \hat{e}_{k}[1]\right]
$$

has $2 k$ zero crossings, when the above convention of zero crossing counting is exercised.

$$
\begin{aligned}
& \text { [ [ } e[0]] \\
& =\frac{1}{\sqrt{2}}[\underbrace{\sqrt{2} \hat{e}_{k}[0] \hat{e}_{k}(1) \ldots \hat{e}_{k}[r]}_{k \text { zero crossings }}: \underbrace{\vdots \hat{e}_{k}[r] \ldots \hat{e}_{k}[1] \sqrt{2} \hat{e}_{k}[0]}_{k \text { zero crossings }}
\end{aligned}
$$

where $N=2 r+1$. The same result can also be shown for $N=2 r$.

Similarly, odd eigenvectors of $\mathbf{S}$ are derived from the eigenvectors of $\mathrm{Od}$ by zero padding and transformation: $\mathbf{o}=\mathbf{P}\left[0 \ldots 0 \mid \hat{\mathbf{o}}_{k}^{T}\right]^{T}$. It can be further shown that the odd eigenvector $\mathbf{o}$ derived from the eigenvector $\hat{\mathbf{o}}_{k}$ of $\mathbf{O d}$ with $k$ zero crossings is an eigenvector of $\mathbf{S}$ with $2 k+1$ zero crossings $(0 \leq k \leq\lfloor(N-3) / 2\rfloor)[48]$.

This procedure not only enables us to accurately determine the number of zero crossings but also demonstrates that each of the eigenvectors of $\mathbf{S}$ has a different number of zero crossings so that each vector can be assigned an index equal to its number of zero crossing. The index number $k$ spans different ranges, depending on the parity of $N$, that is, $k=\{0, \ldots, N-1\}$ for odd $N$ and $k=\{0, \ldots, N-2, N\}$ for even $N$.

A numerical comparison of the continuous and discrete Hermite-Gaussians is presented in Fig. 1. A comparison of the known properties of discrete and continuous Hermite-Gaussians is presented in Table I. These completely analogous properties strengthen our belief that discrete time counterparts of the other properties of continuous time Hermite-Gaussians can be obtained.

In conclusion, there is a well-defined procedure for finding and ordering the common eigenvector set of the matrix $\mathbf{S}$ and the DFT matrix, such that the $n$th member of this eigenvector 

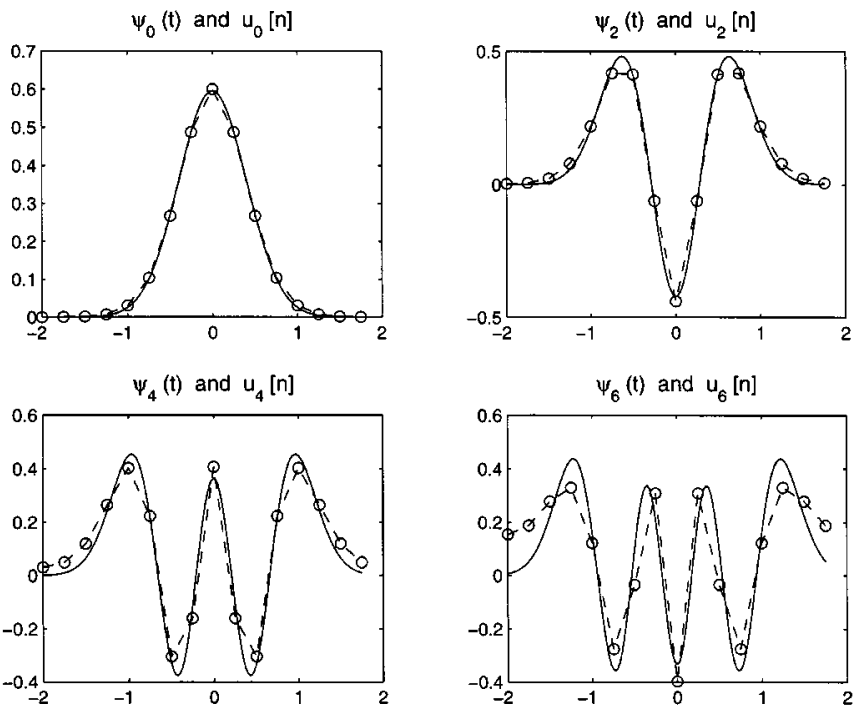

Fig. 1. Comparison of the $\{0,2,4,6\}$ th continuous Hermite-Gaussian functions with the corresponding eigenvectors of the $\mathbf{S}$ matrix $(N=16)$.

set has $n$ zero crossings and is even or odd according to whether $n$ is even or odd.

\section{Definition of the Discrete Fractional Fourier Transform}

The definition of the discrete FRT can now be given as

$$
\mathbf{F}^{a}[m, n]=\sum_{k=0, k \neq\left(N-1+(N)_{2}\right)}^{N} u_{k}[m] e^{-j \frac{\pi}{2} k a} u_{k}[n]
$$

where $u_{k}[n]$ is the $k$ th discrete Hermite-Gaussian function (the eigenvector of $\mathbf{S}$ with $k$ zero crossings) and $(N)_{2} \equiv N \bmod 2$. The peculiar range of the summation is due to the fact that there does not exist an eigenvector with $N-1$ or $N$ zero crossings when $N$ is even or odd, respectively. This index skipping is related to the similarly peculiar eigenvalue multiplicity of the DFT matrix, which is given in Table II.

Some of the properties of the discrete FRT are summarized in Table III.

The matrix $\mathbf{S}+4 \mathbf{I}$ was previously employed in [38]. In [1], Pei and Yeh have observed the visual similarity between the eigenvectors of $\mathbf{S}+4 \mathbf{I}$ and the Hermite-Gaussian functions, noting that it can form the basis of the definition of the discrete fractional Fourier transform. In this paper, we showed why the matrices $\mathbf{S}+4 \mathbf{I}$ or $\mathbf{S}$ have eigenvectors similar to the Hermite-Gaussians, proved the uniqueness of these eigenvectors, and presented a precise method of indexing them in one-to-one correspondence with the Hermite-Gaussians. In the next section, we will find a sequence of matrices $\mathbf{S}_{2 k}$, which provide even finer approximations to the Hermite-Gaussian functions.

The overall procedure underlying the generation of the ath-order discrete FRT matrix is summarized in Table IV.

\section{IV. $\mathbf{S}_{2 k}$ MATRICES}

In Section III, we wrote a difference equation constituting a first-order approximation to the differential equation defining the Hermite-Gaussians. In this section, we will write higher order difference equations that provide better approximations.
TABLE I

COMPARISON OF THE DISCRETE HERMITE-GAUSSIANS WITH THE CONTINUOUS HERMITE-GAUSSIANS

\begin{tabular}{l} 
The continuous Hermite-Gaussians \\
\hline 1. satisfy a generating differential equation. \\
2. are eigenfunctions of the continuous Fourier transform. \\
3. form a complete and orthogonal set in $\mathcal{L}_{2}$. \\
4. can be ordered by their number of zeros. \\
5. The continuous Hermite-Gaussian with $\mathrm{k}$ zeros has the eigenvalue $e^{-j \frac{\pi k}{2}}$ \\
The discrete Hermite-Gaussians \\
1. satisfy a generating difference equation. \\
2. are eigenvectors of the discrete Fourier transform. \\
3. form a complete and orthogonal set in $\mathcal{R}^{N}$. \\
4. can be ordered by their number of zero-crossings. \\
5. The discrete Hermite-Gaussian with $k$ zero-crossings has the eigenvalue \\
e $e^{-j \frac{\pi k}{2}}$ under the discrete Fourier transform operation.
\end{tabular}

TABLE II EIGENVALUE MultiPliCity OF DFT MATRICE

\begin{tabular}{c|c|c|c|c}
\hline$N$ & 1 & $-j$ & -1 & $j$ \\
\hline $4 m$ & $m+1$ & $m$ & $m$ & $m-1$ \\
\hline $4 m+1$ & $m+1$ & $m$ & $m$ & $m$ \\
\hline $4 m+2$ & $m+1$ & $m$ & $m+1$ & $m$ \\
\hline $4 m+3$ & $m+1$ & $m+1$ & $m+1$ & $m$ \\
\hline
\end{tabular}

TABLE III

Properties OF THE Discrete FraCtIONAL FOURIER TRANSFORM

\begin{tabular}{cccc}
\hline 0 & $f[n]$ & $\stackrel{a}{\longleftrightarrow}$ & $f_{a}[n]$ \\
\hline 1 & $f[n]+g[n]$ & $\stackrel{a}{\longleftrightarrow}$ & $f_{a}[n]+g_{a}[n]$ \\
\hline 2 & $f_{a}[n]$ & $\stackrel{b}{\longleftrightarrow}$ & $f_{a+b}[n]$ \\
\hline 3 & $f[n]$ & $\stackrel{a=1}{\longleftrightarrow}$ & $D F T\{f[n]\}$ \\
\hline 4 & $f[-n]$ & $\stackrel{a}{\longleftrightarrow}$ & $f_{a}[-n]$ \\
\hline 5 & $f^{*}[n]$ & $\stackrel{a}{\longleftrightarrow}$ & $f_{-a}^{*}[n]$ \\
\hline 6 & $E v e n\{f[n]\}$ & $\stackrel{a}{\longleftrightarrow}$ & $\operatorname{Even}\left\{f_{a}[n]\right\}$ \\
\hline 7 & Odd $\{f[n]\}$ & $\stackrel{a}{\longleftrightarrow}$ & $O d d\left\{f_{a}[n]\right\}$ \\
\hline 8 & $\sum_{n=0}^{N-1}|f[n]|^{2}$ & $=$ & $\sum_{n=0}^{N-1}\left|f_{a}[n]\right|^{2}$ \\
\hline & & &
\end{tabular}

TABLE IV

GENERATION OF $\mathbf{F}^{a}$ MATRIX

1 Generate matrices $\mathbf{S}$ and $\mathbf{P}$.

2 Generate the Ev and Od matrices from (19).

3 Find the eigenvectors/eigenvalues of Ev and Od.

4 Sort the eigenvectors of Ev (Od) in the descending order of eigenvalues of Ev (Od) and denote the sorted eigenvectors as $\mathbf{e}_{\mathbf{k}}\left(\boldsymbol{o}_{\mathbf{k}}\right)$.

$5 \quad$ Let $\mathbf{u}_{2 \mathbf{k}}[n]=\mathbf{P}\left[\mathbf{e}_{\mathbf{k}}{ }^{T} \mid 0 \ldots 0\right]^{T}$. Let $\mathbf{u}_{\mathbf{2 k + 1}}[n]=\mathbf{P}\left[0 \ldots 0 \mid \mathrm{o}_{\mathbf{k}}^{T}\right]^{T}$.

6 Define $\mathbf{F}^{a}[m, n]=\sum_{k \in \mathcal{M}} u_{k}[m] e^{-j \frac{\pi}{2} k a} u_{k}[n]$, $\mathcal{M}=\left\{0, \ldots, N-2,\left(N-(N)_{2}\right)\right\}$ 
If we denote the $O\left(h^{2 k}\right)$ approximation to $\mathcal{S}$ as $\mathbf{S}_{2 k}$, the matrix $\mathbf{S}$ appearing in the previous section can then be renamed as $\mathbf{S}_{2}$ within this general setting of approximation matrices. As far as an a priori discrete theory is concerned, these higher order approximations may not be of special interest since the lowest order approximation leads to the purest and simplest definition of the discrete fractional Fourier transform. Nevertheless, these approximations may be of use when it is desired to simulate continuous transforms very accurately.

We first derive the higher order approximations to the second derivative operator.

Theorem 4: The $O\left(h^{2 k}\right)$ approximation of $\mathcal{D}^{2}$ can be expressed as

$$
\tilde{\mathcal{D}}_{2 k}=\frac{1}{h^{2}} \sum_{m=1}^{k}(-1)^{m-1} \frac{2[(m-1) !]^{2}}{(2 m) !}\left(h^{2} \tilde{\mathcal{D}}^{2}\right)^{m} .
$$

Proof of the theorem is given in [48].

As an example, we examine the $O\left(h^{4}\right)$ approximation of $\mathcal{D}^{2}$ :

$$
\begin{aligned}
\left(\tilde{\mathcal{D}}^{2}{ }_{4} f(n h)=\right. & \frac{1}{h^{2}}\left[h^{2} \tilde{\mathcal{D}}^{2}-\frac{1}{12}\left(h^{2} \tilde{\mathcal{D}}^{2}\right)^{2}\right] f(n h) \\
= & \frac{1}{h^{2}}\left[-\frac{1}{12} f[n+2]+\frac{4}{3} f[n+1]-\frac{5}{2} f[n]\right. \\
& \left.+\frac{4}{3} f[n-1]-\frac{1}{12} f[n-2]\right]
\end{aligned}
$$

where $f[n]=f(n h)$, and the definition of $\tilde{\mathcal{D}}^{2}$ is given in (11).

As in Section III-A, replacing $\mathcal{D}^{2}$ with $(\tilde{\mathcal{D}})_{2 k}$ in (6) and proceeding similarly, we may ultimately reach the higher order approximation matrices $\mathbf{S}_{2 k}$. The eigenvectors of these matrices will be better approximations to the Hermite-Gaussian functions. For example, the $7 \times 7 \mathrm{~S}_{4}$ matrix is given by

$$
\mathbf{S}_{4}=\left[\begin{array}{ccccccc}
C_{0} & \frac{4}{3} & -\frac{1}{12} & 0 & 0 & -\frac{1}{12} & \frac{4}{3} \\
\frac{4}{3} & C_{1} & \frac{4}{3} & -\frac{1}{12} & 0 & 0 & -\frac{1}{12} \\
-\frac{1}{12} & \frac{4}{3} & C_{2} & \frac{4}{3} & -\frac{1}{12} & 0 & 0 \\
0 & -\frac{1}{12} & \frac{4}{3} & C_{3} & \frac{4}{3} & -\frac{1}{12} & 0 \\
0 & 0 & -\frac{1}{12} & \frac{4}{3} & C_{4} & \frac{4}{3} & -\frac{1}{12} \\
-\frac{1}{12} & 0 & 0 & -\frac{1}{12} & \frac{4}{3} & C_{5} & \frac{4}{3} \\
\frac{4}{3} & -\frac{1}{12} & 0 & 0 & -\frac{1}{12} & \frac{4}{3} & C_{6}
\end{array}\right]
$$

where $C_{k}=2(-1 / 12 \cos (2 \pi / N(2 k))+4 / 3 \cos (2 \pi / N k)-$ $5 / 4)$.

It is also possible to show that the eigenvectors of the matrix $\mathbf{S}_{2 k}$ are also eigenvectors of the DFT matrix by slightly modifying the proof of Theorem 2. Unfortunately, the proof of uniqueness of the eigenvectors of $\mathbf{S}_{2 k}$ and the method of ordering them cannot be generalized since $\mathbf{S}_{2 k}$ matrices are not tridiagonal. Nevertheless, extensive numerical simulations show that the procedure for defining the discrete Hermite-Gaussians through $\mathbf{S}_{2}$ does generalize for higher order matrices. Further work is needed to provide rigorous statements.

\section{NUMERICAL COMPARISON}

In this section, we will compare the continuous fractional Fourier transform of a rectangular pulse with the discrete frac- tional Fourier transform of the sampled version of the same function. The rectangular pulse is defined as

$$
f(t)= \begin{cases}1, & |t|<1 \\ 0, & \text { otherwise }\end{cases}
$$

The continuous FRT of the pulse is calculated using the numerical integration routines of MATLAB with a tolerance of 0.001. The discrete FRT transform of the pulse is calculated by transforming the $N$ samples of the pulse function in the interval $[-\sqrt{N} / 2, \sqrt{N} / 2]$. From Fig. 2, we can see that the discrete transform output is a fairly good approximation of the continuous transform. Similar results have been obtained with many other functions.

\section{CONCLUSION}

We have presented a definition of the discrete FRT that exactly satisfies the essential operational properties of the continuous fractional Fourier transform. This definition sets the stage for a self-consistent discrete theory of the fractional Fourier transformation and makes possible a priori discrete formulations in applications.

As a by product, we obtained the discrete counterparts of the Hermite-Gaussian functions. We believe that the discrete counterparts of the multitude of operational properties for the Hermite-Gaussian functions, such as recurrence relations, differentiation properties, etc., can be derived by methods similar to those in Section III. Likewise, we believe the discrete counterparts of the many interesting and useful properties of the continuous fractional transform will be established.

Our method of derivation closely resembles the continuous definition originally given in [6] and [7]. In these papers, the continuous FRT is defined through spectral expansion. It was later noticed that Namias had reached the integral kernel of the FRT from its spectral expansion using certain identities and formulas for the Hermite-Gaussian functions [3]. To reach the closed-form definition for the discrete FRT, we need similar identities and formulas for the discrete Hermite-Gaussians defined in this paper. Further research on the difference equation yielding the discrete Hermite-Gaussians may provide some results leading to the closed-form definition of the discrete FRT.

Although not touched on in this paper, there exists another way of interpreting the continuous $\mathcal{S}$ operator. Readers with a physics background may have noticed that $\mathcal{S}$ is actually the Hamiltonian of the harmonic oscillator, which is a system of fundamental importance in mechanics. The matrix $\mathbf{S}$ introduced in this paper may be proposed as the difference analog of the continuous harmonic oscillator Hamiltonian and may lead to further interesting connections [49]. We may refer to [36], [51] for another discretization of the harmonic oscillator Hamiltonian.

We have already mentioned that the $O(N \log N)$ algorithm presented in [34] can be utilized for fast computation in most applications. For example, in the linear-system-approximation application in [17] and [18], we can implement the fractional Fourier transform stages with this fast algorithm. However, it would be preferable to have a fast algorithm that exactly com- 

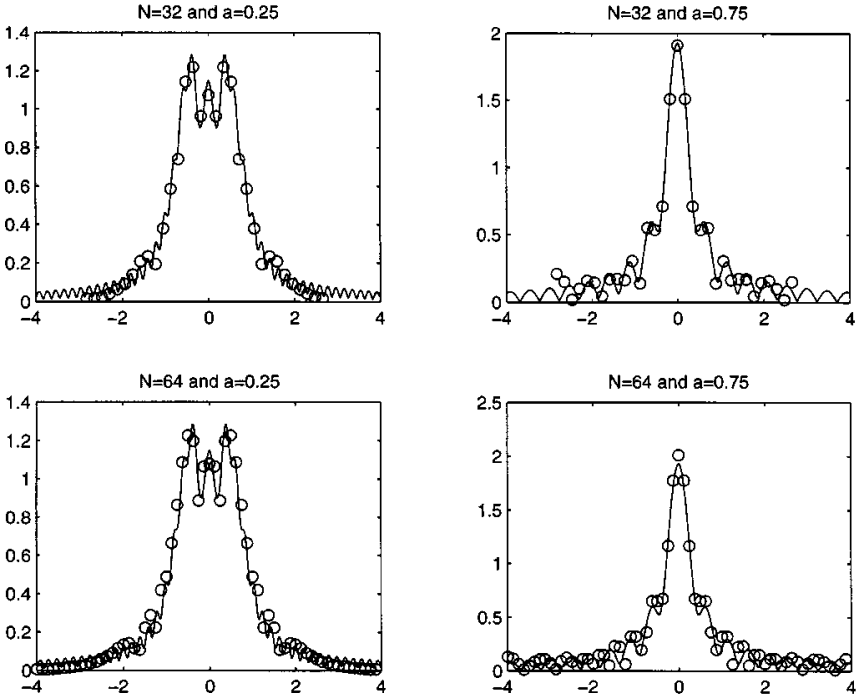

Fig. 2. FRT of the rectangle function calculated through numerical integration (solid curve) and through the discrete FRT matrix (circles). The transform order $a$ and matrix dimension $N$ is indicated in the title of each plot.

putes the product of the fractional Fourier transform matrix defined here, with an arbitrary vector. We are confident that such an algorithm will emerge soon.

From the spectral expansion of the continuous and discrete fractional Fourier transform, it is intuitively clear that the discrete transform approaches the continuous transform as the finite step size $h$ approaches 0 or, equivalently, as $N$ approaches $\infty$. A rigorous statement of this remains to be established. Nevertheless, certain forms of convergence results for discrete Hermite-Gaussians have been presented in [50].

One of the most interesting avenues for future research is the establishment of the relationship of the discrete fractional Fourier transform with the discrete Wigner distribution. We might expect the study of the relationship of the Wigner distribution with the fractional Fourier transform to contribute to the establishment of a definitive definition of the discrete Wigner distribution, leading to a consolidation of the theory of discrete time-frequency analysis.

Last, since the DFT is closely related to many other transforms such as the discrete cosine, Haar, Hadamard, Hartley, etc., generalizations of these transforms to fractional orders and their applications can be accomplished in the near future.

\section{REFERENCES}

[1] S. C. Pei and M. H. Yeh, "Improved discrete fractional fourier transform," Opt. Lett., vol. 22, pp. 1047-1049, 1997.

[2] S. C. Pei, C. C. Tseng, M. H. Yeh, and J. J. Shyu, "Discrete fractional Hartley and Fourier transforms," IEEE Trans. Circuits Syst. II, vol. 45, pp. 665-675, 1998

[3] V. Namias, "The fractional order Fourier transform and its application to quantum mechanics," J. Inst. Math. Appl., vol. 25, pp. 241-265, 1980.

[4] A. C. McBride and F. H. Kerr, "On Namias's fractional Fourier transform," IMA J. Appl. Math., vol. 39, pp. 159-175, 1987.

[5] H. M. Ozaktas, M. A. Kutay, and D. Mendlovic, "Introduction to the fractional Fourier transform and its applications," in Advances in Imaging Electronics and Physics. New York: Academic, 1999, ch. 4.
[6] D. Mendlovic and H. M. Ozaktas, "Fractional Fourier transforms and their optical implementation 1," J. Opt. Soc. Amer. A., vol. 10, pp. 1875-1881, 1993.

[7] H. M. Ozaktas and D. Mendlovic, "Fractional Fourier transforms and their optical implementation 2," J. Opt. Soc. Amer. A., vol. 10, pp. 2522-2531, 1994.

[8] H. M. Ozaktas, B. Barshan, D. Mendlovic, and L. Onural, "Convolution, filtering, and multiplexing in fractional Fourier domains and their relation to chirp and wavelet transforms," J. Opt. Soc. Amer. A., vol. 11, pp. 547-559, 1994.

[9] A. W. Lohmann, "Image rotation, Wigner rotation, and the fractional Fourier transform," J. Opt. Soc. Amer. A., vol. 10, pp. 2181-2186, 1993.

[10] A. W. Lohmann and B. H. Soffer, "Relationships between the RadonWigner and fractional Fourier transforms," J. Opt. Soc. Amer. A., vol. 11, pp. 1798-1811, 1994.

[11] D. Mustard, "The fractional Fourier transform and the Wigner distribution," J. Austral. Math. Soc. B-Appl. Math., vol. 38, pp. 209-219, 1996.

[12] L. B. Almeida, "The fractional Fourier transform and time-frequency representations," IEEE Trans. Signal Processing, vol. 42, pp. 3084-3091, Nov. 1994.

[13] M. A. Kutay, H. M. Ozaktas, L. Onural, and O. Arıkan, "Optimal filtering in fractional Fourier domains," in Proc. IEEE Int. Conf. Acoust., Speech, Signal Process., vol. 2, 1995, pp. 937-941.

[14] M. A. Kutay, H. M. Ozaktas, O. Arikan, and L. Onural, "Optimal filtering in fractional Fourier domains," IEEE Trans. Signal Processing, vol. 45, pp. 1129-1143, July 1997.

[15] — "Optimal image restoration with the fractional Fourier transform," J. Opt. Soc. Amer. A., vol. 15, pp. 825-833, 1998.

[16] Z. Zalevsky and D. Mendlovic, "Fractional Wiener filter," Appl. Opt., vol. 35, pp. 3930-3936, 1996.

[17] M. F. Erden and H. M. Ozaktas, "Synthesis of general linear systems with repeated filtering in consecutive fractional Fourier domains," $J$. Opt. Soc. Amer. A., vol. 15, pp. 1647-1657, 1998.

[18] M. A. Kutay, M. F. Erden, H. M. Ozaktas, O. Arıkan, Ö. Güleryüz, and Ç. Candan, "Space-bandwidth efficient realizations of linear systems," Opt. Lett., vol. 23, pp. 1069-1071, 1998.

[19] — - "Cost-efficient approximation of linear systems with repeated and multi-channel filtering configurations," in Proc. IEEE Int. Conf. Acoust. Speech Signal Process., vol. 6, 1998, pp. 3433-3437.

[20] M. F. Erden, M. A. Kutay, and H. M. Ozaktas, "Repeated filtering in consecutive fractional Fourier domains and its application to signal restoration," IEEE Trans. Signal Processing, vol. 47, pp. 1468-1462, May 1999.

[21] M. A. Kutay, H. Özaktaş, M. F. Erden, H. M. Ozaktas, and O. Arıkan, "Solution and cost analysis of general multi-channel and multi-stage filtering circuits," in Proc. IEEE SP Symp. Time-Freq. Anal., vol. 1, 1998, pp. 481-484.

[22] H. M. Ozaktas, N. Erkaya, and M. A. Kutay, "Effect of fractional Fourier transformation on time-frequency distributions belonging to the cohen class," IEEE Signal Processing Lett., vol. 40, pp. 40-41, Feb. 1996.

[23] O. Akay and G. F. Boudreaux-Bartels, "Joint fractional representations," in Proc. IEEE SP Symp. Time-Freq. Anal., vol. 1, 1998, pp. 417-420.

[24] H. M. Ozaktas and D. Mendlovic, "Fractional Fourier optics," J. Opt. Soc. Amer. A., vol. 12, pp. 743-751, 1995.

[25] H. M. Ozaktas and M. F. Erden, "Relationships among ray optical, Gaussian beam, and fractional Fourier transform descriptions of first-order optical systems," Opt. Commun., vol. 143, pp. 75-86, 1997.

[26] D. Mendlovic, Z. Zalevsky, and H. M. Ozaktas, "The applications of the fractional Fourier transform to optical pattern recognition," in Optical Pattern Recognition. New York: Academic, 1998, ch. 3.

[27] A. I. Zayed, "On the relationship between the Fourier and fractional Fourier transforms," IEEE Signal Processing Lett., vol. 3, pp. 310-311, Nov. 1996.

[28] X. Xia, "On bandlimited signals with fractional Fourier transform," IEEE Signal Processing Lett., vol. 3, pp. 72-74, Mar. 1996.

[29] L. B. Almeida, "Product and convolution theorems for the fractional Fourier transform," IEEE Signal Processing Lett., vol. 4, pp. 15-17, Jan. 1997.

[30] A. I. Zayed, "Convolution and product theorem for the fractional Fourier transform," IEEE Signal Processing Lett., vol. 5, pp. 101-103, Apr. 1998 . 
[31] — "Hilbert transform associated with the fractional Fourier transform," IEEE Signal Processing Lett., vol. 5, pp. 206-208, July 1998.

[32] M. J. Bastiaans and A. J. van Leest, "From the rectangular to the quincunx gabor lattice via fractional Fourier transformation," IEEE Signal Processing Lett., vol. 5, pp. 203-205, July 1998.

[33] A. Sahin, M. A. Kutay, and H. M. Ozaktas, "Non-separable two-dimensional fractional Fourier transform,” Appl. Opt., vol. 37, pp. 5444-5453, 1998.

[34] H. M. Ozaktas, O. Arıkan, M. A. Kutay, and G. Bozdag̃i, "Digital computation of the fractional Fourier transform," IEEE Trans. Signal Processing, vol. 44, pp. 2141-2150, Sept. 1996.

[35] O. Arıkan, M. A. Kutay, H. M. Ozaktas, and Ö. K. Akdemir, "Discrete fractional Fourier transformation," Proc. IEEE SP Symp. TimeFreq. Anal., vol. 4, pp. 205-207, 1996.

[36] N. M. Atakishiyev and K. B. Wolf, "Fractional Fourier-Kravchuk transform," J. Opt. Soc. Amer. A., vol. 14, pp. 1467-1477, 1997.

[37] M. S. Richman, T. W. Parks, and R. G. Shenoy, "Understanding discrete rotations," in Proc. IEEE Int. Conf. Acoust. Speech Signal Process., 1997.

[38] B. W. Dickinson and K. Steiglitz, "Eigenvectors and functions of the discrete Fourier transform," IEEE Trans. Acoust., Speech, Signal Processing, vol. ASSP-30, pp. 25-31, Jan. 1982.

[39] B. Santhanam and J. H. McClellan, "The discrete rotational Fourier transform," IEEE Trans. Signal Processing, vol. 44, pp. 994-998, Apr. 1996.

[40] G. Cariolaro, T. Erseghe, P. Kraniauskas, and N. Laurenti, "Multiplicity of fractional Fourier transforms and their relations," IEEE Trans. Signal Processing, vol. 48, pp. 227-241, Jan. 2000.

[41] J. H. McClellan and T. W. Parks, "Eigenvalue and eigenvector decomposition of the discrete Fourier transform," IEEE Trans. Audio Electroacoust., vol. AU-20, pp. 66-74, 1972.

[42] P. Beckmann, Orthogonal Polynomials for Engineers and Physicists. New York: Golem, 1973.

[43] G. Birkhoff and G. C. Rota, Ordinary Differential Equations. New York: Wiley, 1989.

[44] K. B. Wolf, "Construction and properties of canonical transforms," in Integral Transforms in Science and Engineering. New York: Plenum, 1979, ch. 9.

[45] J. H. Wilkinson, The Algebraic Eigenvalue Problem. Oxford, U.K.: Oxford, 1988.

[46] F. Hildebrand, Finite-Difference Equations and Simulations. Englewood Cliffs, NJ: Prentice-Hall, 1968.

[47] S. N. Elaydi, An Introduction to Difference Equations. New York: Springer, 1996.

[48] Ç. Candan, "The Discrete Fractional Fourier Transform," M.S. thesis, Bilkent Univ., Ankara, Turkey, 1998

[49] L. J. Barker, Ç. Candan, T. Hakiog̃lu, M. A. Kutay, and H. M. Ozaktas, "The harmonic oscillator, Harper's equation and the discrete fractional Fourier transform," J. Phys. A.: Math., vol. 33, pp. 2209-2222, Mar. 2000.

[50] L. J. Barker, "The discrete fractional Fourier transform and Harper's equation," preprint.

[51] N. M. Atakishiyev and S. K. Suslov, "Difference analogs of the harmonic oscillator," Theor. Math. Phys., vol. 85, pp. 1055-1062, 1991.

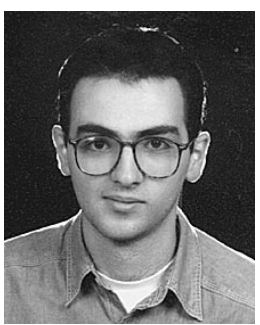

Çag̃atay Candan (S'99) was born in Ankara, Turkey. He received the B.S. degree from Middle East Technical University, Ankara, in 1996 and the M.S. degree from Bilkent University, Ankara, in 1998 , both in electrical engineering. He is currently pursuing the Ph.D. degree at the Georgia Institute of Technology, Atlanta, in the area of information theory and image processing.

His research interests include fractional Fourier transforms, time-frequency analysis, and signal processing theory.

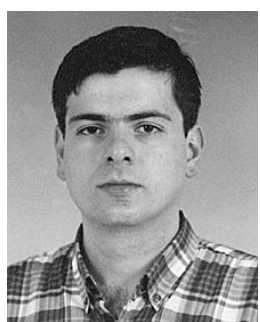

M. Alper Kutay (M'99) was born in Konya, Turkey, in 1972. He received the B.S., M.S., and Ph.D. degrees, all in electrical and electronics engineering, from Bilkent University, Ankara, Turkey in 1993, 1995, and 1999, respectively.

During his graduate studies, he was a Teaching and Research Assistant. He is currently with the Communications and Signal Processing Laboratory, Drexel University, Philadelphia, PA. His current research areas include time-frequency representations, fractional Fourier transform, time-varying filtering, and biomedical signal characterization and processing.

Dr. Kutay was awarded third place in the 1994 IEEE region 8 student paper contest for his work entitled "An adaptive speckle suppression filter for medical ultrasonic imaging."

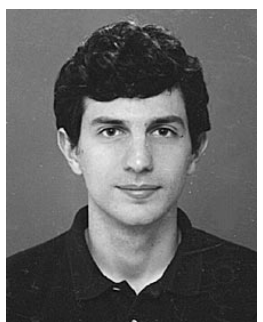

Haldun M. Ozaktas received the B.S. degree from Middle East Technical University, Ankara, Turkey, in 1987 and the Ph.D. degree from Stanford University, Stanford, CA, in 1991.

During his graduate studies, he was a Research Assistant to J. W. Goodman. He joined Bilkent University, Ankara, in 1991, where he is presently Professor of electrical engineering. In 1992, he was with A. W. Lohmann at the University of Erlangen-Nürnberg, Nürnberg, Germany, as an Alexander von Humboldt Foundation Postdoctoral Fellow. Over the summer of 1994, he worked as a Consultant for D. A. B. Miller at Bell Laboratories, Murray Hill, NJ. He is the author of over 60 refereed journal articles, several book chapters, and over 50 conference presentations and papers. His academic interests include optical information processing, signal and image processing, and optoelectronic and optically interconnected computing systems.

Dr. Ozaktas was the recipient of the International Commission for the 1998 International Prize in Optics (jointly with D. Mendlovic). 